\title{
Optimal Transseptal Puncture Location for Robot-Assisted Left Atrial Catheter Ablation
}

\author{
Jagadeesan Jayender ${ }^{1}$, Rajni V. Patel ${ }^{2}$, Gregory F. Michaud ${ }^{3}$, \\ and Nobuhiko Hata ${ }^{1}$ \\ 1 Department of Radiology, Harvard Medical School, \\ Brigham and Women's Hospital, USA \\ jayender@bwh.harvard.edu, hata@bwh.harvard.edu
}

2 Department of Electrical and Computer Engineering and Department of Surgery

The University of Western Ontario,

Canadian Surgical Technologies and Advanced Robotics (CSTAR), Canada

rajni.patel@lhsc.on.ca

3 Department of Cardiology, Harvard Medical School,

Brigham and Women's Hospital, USA

gfmichaud@partners.org.

\begin{abstract}
The preferred method of treatment for Atrial Fibrillation (AF) is by catheter ablation wherein a catheter is guided into the left atrium through a transseptal puncture. However, the transseptal puncture constrains the catheter, thereby limiting its maneuverability and increasing the difficulty in reaching various locations in the left atrium. In this paper, we address the problem of choosing the optimal transseptal puncture location for performing cardiac ablation to obtain maximum maneuverability of the catheter. We have employed an optimization algorithm to maximize the Global Isotropy Index (GII) to evaluate the optimal transseptal puncture location. As part of this algorithm, a novel kinematic model for the catheter has been developed based on a continuum robot model. Preoperative MR/CT images of the heart are segmented using the open source image-guided therapy software, Slicer 3, to obtain models of the left atrium and septal wall. These models are input to the optimization algorithm to evaluate the optimal transseptal puncture location. Simulation results for the optimization algorithm are presented in this paper.
\end{abstract}

\section{Introduction}

Atrial fibrillation (AF) is a type of arrhythmia where the atria beat irregularly and out of coordination with the ventricles. When rate and rhythm control drugs are ineffective in patients suffering from AF, catheter ablation is performed as a standard method of treatment. More recently, a robotics based master-slave approach for performing cardiac ablation has been introduced. At our institution, we have direct clinical experience with the Sensei robot from Hansen Medical Inc. 1]. Although the robot is capable of precise catheter motions and maintaining stable contact with the heart wall, the clinician often encounters 

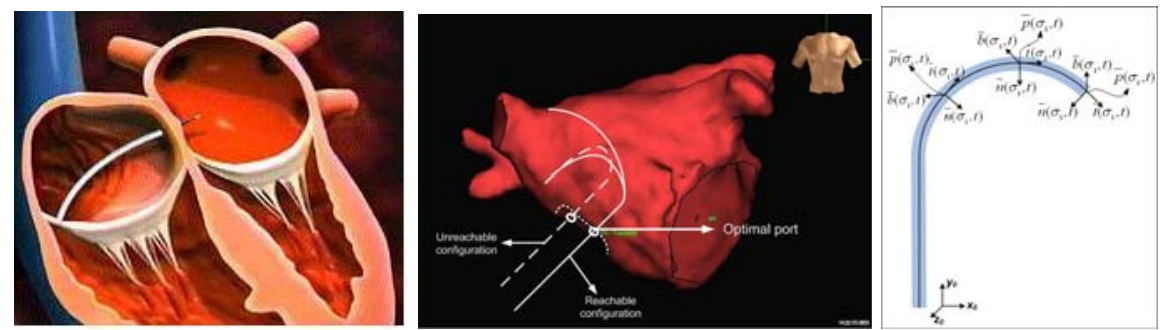

Fig. 1. (a) Catheter entering the LA through the transseptal puncture, (b) Difficulty in maneuvering the catheter due to improper transseptal puncture location, (c) Model for the catheter

difficulty in maneuvering the catheter and approaching the desired point on the heart at the required angle. The lack of dexterity of the catheter arises from the constraint imposed on the motion of the catheter due to the transseptal puncture [2, see Figure 1(a). The interatrial septal wall effectively acts like a Remote Center of Motion (RCM) about which the catheter is manipulated. This can severely affect the dexterity and maneuverability of the catheter (see Figure 1(b)), thereby affecting the clinician's performance. In addition, there is also danger of perforating the aortic arch with catastrophic results due to accidental needle puncture [3]. Therefore, it is of prime importance for the clinician to puncture the transseptal wall at an optimal point to maximize the dexterity of the catheter in the left atrium while minimizing the possibility of puncturing the aortic arch. In this paper, we develop a computational algorithm to determine the optimal transseptal puncture location for performing cardiac ablation. Once determined, the puncture location will be registered to the Intracardiac Echo (ICE) image so that the clinician can guide the needle in real-time to the optimal puncture location to obtain maximum maneuverability of the catheter, while avoiding puncturing of the aorta. The problem of choosing the location of the transseptal puncture bears similarity to the problem of choosing the ports on the thoracic cavity for insertion of laparoscopic tools and endoscopes to perform Robot-Assisted Minimally Invasive Surgery (RA-MIS). Several researchers have studied the problem of choosing the optimal ports to maximize the performance of the robot (and surgeon) to perform RA-MIS [4]-7]. In this paper we have adopted the Global Isotropy Index (GII) [8], [5] to define the performance measure for quantifying the dexterity of the catheter within the left atrium. We have developed an optimization algorithm to maximize the GII for efficiently maneuvering the catheter in the left atrium.

\section{Modeling of Catheter}

The catheter is considered as being made up of infinitesimal rigid links along a backbone curve. The backbone curve is defined in terms of the Frenet-Serret frame. The curve is represented in the parametric form, $\bar{x}=\bar{x}(s, t)$, where $s$ is the 
parameter which represents the curve length and $t$ is the time. The Frenet-Serret frame is defined at each point $\sigma(s, t)$ along the backbone curve and consists of the tangent $\bar{t}(s, t)$, normal $\bar{n}(s, t)$ and binormal $\bar{b}(s, t)$ vector at point $\sigma(s, t)$, as shown in Figure 1(c). At any point $\sigma$ along the curve $\bar{x}(s, t)$, the local frame can be defined as $\Phi(\sigma, t)$. In terms of the Euler angles, the frame ${ }^{0} \Phi(\sigma, t)$ can be defined with respect to the base frame ${ }^{0} \Phi(0, t)$ as a rotation of $\alpha$ about $\bar{b}(\sigma)$ and $\beta$ about $\bar{n}(\sigma)$ vector. Also the angles $\alpha$ and $\beta$ can be defined in terms of the curvature and torsion as follows

$$
\alpha=\int_{0}^{s} \varsigma(\sigma, t) d \sigma \quad \beta=\int_{0}^{s} \tau(\sigma, t) d \sigma
$$

In this case, we assume that the curvature $\varsigma$ and torsion $\tau$ are constant. Therefore, ${ }^{0} \Phi(\sigma, t)$ can be written as

$$
\begin{array}{r}
{ }^{0} \Phi(\sigma, t)=\operatorname{Rot}(\bar{b}, \alpha) \operatorname{Rot}(\bar{n}, \beta) \text { i.e., } \\
{ }^{0} \Phi(\sigma)=\left[\begin{array}{ccc}
\cos (\sigma \varsigma) \cos (\sigma \tau) & -\sin (\sigma \varsigma) & \cos (\sigma \varsigma) \sin \sigma \tau \\
\sin (\sigma \varsigma) \cos (\sigma \tau) & \cos (\sigma \varsigma) & \sin (\sigma \varsigma) \sin (\sigma \tau) \\
-\sin (\sigma \tau) & 0 & \cos (\sigma \tau)
\end{array}\right]
\end{array}
$$

The position vector $\bar{p}(\sigma, t)$ of a point $\sigma$ on the curve relative to the origin $\bar{p}(0)$ can be computed by integrating infinitesimal curve lengths along the tangent vector. In order words, ${ }^{0} \bar{p}(\sigma)$, which represents the position of a point $\sigma$ on the curve as viewed in the base frame ${ }^{0} \Phi(0)$ is given by

$$
{ }^{0} \bar{p}(\sigma, t)=\int_{0}^{s}{ }^{0} \Phi(\eta, t) \hat{e_{x}} d \eta
$$

Each segment of the catheter within the heart can be considered to consist of two rotational joints and a prismatic joint. The joint angle vector can be written as $\bar{\theta}=\left[\begin{array}{lll}0 & \tau\end{array}\right]^{t}$ and the translational vector can be written as $\bar{d}=\left[\begin{array}{lll}l_{x} & 0 & 0\end{array}\right]^{t}$ The rotational velocity for a joint in the local Frenet-Serret frame with respect to the base frame can be written as

$$
\begin{array}{r}
{ }^{0} \omega(\sigma)=\int_{0}^{\sigma} \overline{\dot{\theta}}^{\sigma} \Phi(\nu) d \nu(\text { for a rotational joint) } \\
{ }^{0} \omega(\sigma)=0 \text { (for a prismatic joint) }
\end{array}
$$

Similarly the translational velocities for the rotational and prismatic joints can be written as

$$
\begin{array}{r}
{ }^{0} v(\sigma)=\int_{0}^{\sigma} \overline{\dot{\theta}}^{\sigma} \Phi(\nu) \times\left({ }^{\sigma} p(\nu, t)-{ }^{\sigma} p(\nu, t)\right) d \nu \text { (for a rotational joint) } \\
{ }^{0} v(\sigma)=\int_{0}^{\sigma} \bar{d}^{\sigma} \Phi(\nu) d \nu \text { (for a prismatic joint) }
\end{array}
$$

Equations (5)-(8) together can be written in a compact form as

$$
\left[\begin{array}{c}
{ }^{0} v(\sigma) \\
{ }^{0} \omega(\sigma)
\end{array}\right]=\int_{0}^{\sigma}\left[\begin{array}{c}
\sigma \\
\Phi(\nu, t)[p(\nu, t)-p(\sigma, t) \times] \Phi(\nu, t) \\
0 \\
\Phi(\nu, t)
\end{array}\right] \bar{A}\left[\begin{array}{c}
\dot{d} \\
\dot{\zeta} \\
\dot{\tau}
\end{array}\right] d \nu
$$


where

$$
\bar{A}=\left[\begin{array}{llllll}
1 & 0 & 0 & 0 & 0 & 0 \\
0 & 0 & 0 & 0 & 0 & 1 \\
0 & 0 & 0 & 0 & 1 & 0
\end{array}\right]^{T} \quad \text { and }[a \times] \triangleq\left[\begin{array}{ccc}
0 & -a_{z} & a_{y} \\
a_{z} & 0 & -a_{x} \\
-a_{y} & a_{x} & 0
\end{array}\right]
$$

Using the standard robotics terminology, the Jacobian operator can be defined as

$$
\mathcal{J}(\sigma, t)=\int_{0}^{\sigma}\left[\begin{array}{cc}
\sigma \Phi(\nu, t)[p(\nu, t)-p(\sigma, t) \times] \Phi(\nu, t) \\
0 & \Phi(\nu, t)
\end{array}\right] \bar{A}(.) d \nu
$$

From (4), we can estimate the position of the catheter with respect to the base coordinates for a given configuration of the catheter. However, for the optimization algorithm, we will assume that the catheter is in contact with a certain point inside the left atrium while being constrained at the transseptal puncture location. Therefore, this gives us the base and end-effector coordinates of the catheter. It is required to solve the inverse kinematics problem to evaluate the configuration of the catheter for the distal end of the catheter to be in contact with a particular point inside the heart. The problem of solving the inverse kinematics of the catheter is formulated as a dynamical problem which requires only the computation of the forward kinematics, as determined by (4). Let us represent the solution of the inverse kinematics problem as $\hat{q}(t)$ corresponding to a trajectory $\hat{x}(t)$ which satisfies the forward kinematics given by (4). Let $e(t)$ represent the error between the desired Cartesian position $\hat{x}(t)$ and the actual Cartesian position obtained from the state variable $q$ of the iteration algorithm. The error dynamics can be written as

$$
\dot{e}(t)=\dot{\hat{x}}(t)-\dot{x}(t)=\dot{\hat{x}}(t)-\mathcal{J} \dot{q}
$$

We choose a purely proportional control law to solve for $\dot{q}$ as given by

$$
\dot{q}=\alpha \mathcal{J}^{T} e
$$

It has been shown in [9] that by choosing a control law as given by (13), the error $e$ is bounded and can be made small with an appropriate choice of $\alpha$, with the added benefit of less computational complexity.

\section{Global Isotropy Index}

The Jacobian matrix relates the end-effector frame velocities $\dot{x}$ and forces $f$ to the corresponding joint rates $\dot{q}$ and torques $\tau$, as given by the following equations

$$
\begin{array}{r}
\dot{x}=\mathcal{J} \dot{q} \\
\tau=\mathcal{J}^{T} f
\end{array}
$$

Condition number $\kappa$ of the Jacobian $\mathcal{J}$ can be considered as the error amplification factor from the joint space to the Cartesian space. Taking the norm on both sides of (14), we obtain

$$
\frac{\|\delta x\|}{\|x\|} \leq\left\|\mathcal{J}^{-1}\right\|\|\mathcal{J}\| \frac{\|\delta q\|}{\|q\|}
$$

The condition number $\kappa$ is, therefore, defined as 10] 


$$
\kappa(J)=\left\|\mathcal{J}^{-1}\right\|\|\mathcal{J}\|=\frac{\bar{\sigma}(J(q))}{\underline{\sigma}(J(q))}
$$

where $\bar{\sigma}$ and $\underline{\sigma}$ represent the maximum and minimum singular values of $\mathcal{J}$. The condition number represents the dexterity of the robot at a specified point and often, the inverse of the condition number is used since the value of $\kappa(J)^{-1} \in$ $[0,1]$. For a robot in a singular configuration, $\underline{\sigma}=0$, implying that for $\kappa(J)^{-1}=0$. The closer $\kappa(J)^{-1}$ or $\kappa(J)$ is to unity, the more accurate and dextrous the robot is. A configuration of the robot is defined as an isotropic configuration, which is the most dextrous configuration for the robot, when the condition number $\kappa(J)$ corresponding to this configuration is unity. Therefore, in order to ensure that the robot (in our case the catheter) is well controlled in all regions of the left atrium, we try to maximize the dexterity or a measure of isotropy. However, it should be noted that the Jacobian is a function of the local position (in turn joint variables), therefore the condition number is a local measure of the dexterity of the robot at any position. The transseptal puncture location should be chosen such that the dexterity of the catheter is maximized at all points in the left atria. Therefore, there is a need to define the performance measure as a global measure rather than a local measure. In [8], the Global Isotropy Index (GII) was proposed to define the overall dexterity of the robot throughout the workspace. The GII is defined as

$$
G I I\left(y_{i}\right)=\frac{\min _{x_{0} \in W_{i}} \underline{\sigma}\left(\mathcal{J}\left(x_{0}\right)\right)}{\max _{x_{1} \in W_{i}} \bar{\sigma}\left(\mathcal{J}\left(x_{1}\right)\right)}
$$

where $W_{i}=W\left(y_{i}\right)=\left\{x_{k}-y_{i}: x_{k} \in W_{a}\right\}, y_{i} \in W_{s}$. For our application, $W_{a} \in \Re^{3}$ is the set of points on the left atrium and $W_{s} \in \Re^{3}$ is the set of points on the septal wall. It should be noted that GII is a global measure as compared to the condition number which is a local measure. In addition, it should also be noted that the GII is related to the inverse of the condition number rather than the condition number itself, in order to restrict the values to the range $[0,1]$. A GII of 1 implies that the catheter is isotropic at every point in the workspace and behaves uniformly in all directions, which is the ideal condition.

Since linear and circumferential lesions may be needed at most points in the left atrium depending on the type of atrial fibrillation (persistant, paroxysmal or permanent), we consider the surgical workspace to be the entire left atrium. The workspace can be further reduced based on the clinician's input in the preoperative stage. The problem is to choose the transseptal puncture location to maximize the dexterity of the catheter while accessing the surgical workspace. The dexterity of the catheter in the surgical workspace is quantified in terms of the GII. The optimization algorithm searches for the point $y_{o p t} \in W_{s}$, which maximizes the GII, corresponding to the optimal location for the transseptal puncture. This location constrains the catheter and can be considered as the base coordinates of the catheter. The distal end of the catheter (considered as the end-effector) touches the points on the left atrium. For each point on the left atrium, the configuration of the catheter is estimated using inverse kinematics 
and the corresponding Jacobian $\mathcal{J}$ and singular values $(\bar{\sigma}, \underline{\sigma})$ are evaluated and provided to the optimization algorithm.

\section{Experimental Results}

A pre-operative CT image was first loaded in Slicer 3. The CT image is a contrast enhanced image consisting of 64 slices. The resolution of each slice is $0.7 \mathrm{~mm} \times 0.7 \mathrm{~mm} \times 0.7 \mathrm{~mm}$. An expert radiologist was given the task of manually segmenting the left atrium, the septal wall and the right atrium. The models were then generated in Slicer 3 and the corresponding VTK file consisting of the data points of the model was created. The file consisting of the coordinates of the left atrium and the septal wall were input to the optimization algorithm. The optimal transseptal puncture location algorithm was implemented using MATLAB on an Intel Core2 Duo 2.00GHz machine with 1GB RAM. Figure2 (a) and (b) show the results of the variation of GII as a function of $x$ and $y$ coordinates respectively. The minimum value of GII was 0.0112 and the maximum value was 0.4362 , a location which provides the best isotropy and in turn dexterity of the catheter. In addition, the GII values were converted to a scalar VIBGYOR color map (red representing low GII and violet representing high GII) corresponding to the points in the VTK file and displayed on the model of the septal wall in Slicer, as shown in Figure 3(a). Since the catheter is constrained to the transseptal puncture point, the catheter loses some dexterity and this can be noticed in the low values of the GII at most points on the septal wall, represented by the red color in Figure 3(a). The mean value of GII on the septal wall was 0.0327 . Figure 3(b) shows the location of the optimal puncture with respect to the left and right atria. Figure 4 (a) shows the mesh plot of the GII on the septal wall. The colorbar reflects the values of the GII at different locations on the septal wall.

In Figure 4(b), we have compared the condition number $\kappa$ for six points on the left atria corresponding to two transseptal puncture locations $S_{1}$ and $S_{2}$. It can be seen that although the GII corresponding to the point $S_{1}$ is larger than that
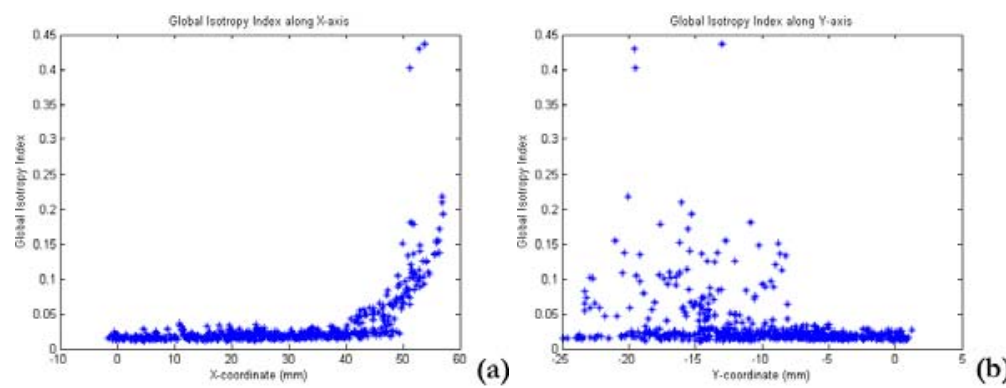

Fig. 2. (a) Graph showing the variation of GII as a function of the x-coordinate, (b) Graph showing the variation of GII as a function of the y-coordinate 


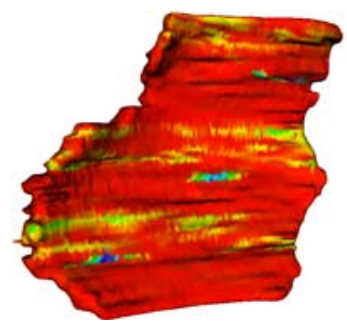

(a)

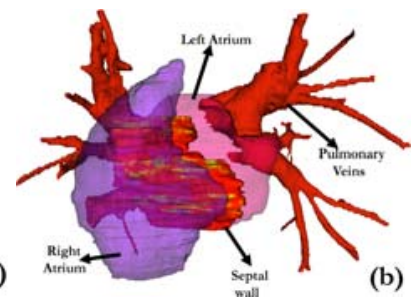

Fig. 3. (a) Magnified view of the septal wall with the VIBGYOR color map representing the GII at various locations. Red represents points with low GII while blue represents points with high GII; (b) Segmented model of the LA, RA, PV and septal wall with the color map representing the GII at different locations.
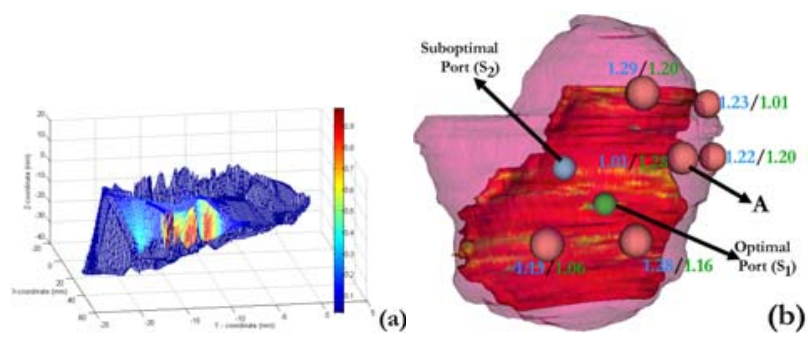

Fig. 4. (a) Mesh plot showing the color map overlaid over the septal wall with colorbar representing the GII, (b) 3D view of 2 points chosen on the septal wall and 6 points on LA. The condition number corresponding to each point is represented as $x_{1} / x_{2}$, where $x_{1}$ corresponds to the condition number of the point assuming that the catheter is passing through the suboptimal port $S_{2}$ and $x_{2}$ corresponds to the condition number of the point assuming that the catheter is passing through the optimal port $S_{1}$.

corresponding to $S_{2}$, the condition number at point $A$ is smaller (1.01) for $S_{2}$ compared to the condition number of 1.28 for $S_{1}$. This implies that the control of the catheter is more accurate at point $A$, if $S_{2}$ is chosen as the transseptal puncture location. As mentioned earlier, GII is a global measure of the dexterity or isotropy of the catheter at all points in the left atria while the condition number is a local measure. It should also be noted that the result of the transseptal puncture algorithm is highly specific to the patient and is dependent on the shape and size of the left atrium, position and number of pulmonary veins and the shape and size of the septal wall.

\section{Conclusion}

The objective of this work was to determine the optimal transseptal puncture location and assist the clinician in guiding the needle in real-time to this location. In this paper, we have developed an optimization algorithm based on the Global 
Isotropy Index (GII) to evaluate the optimal position of the transseptal puncture for left atrial cardiac ablation. As part of this algorithm, we have extended the continuum robot model to describe the kinematics of the catheter within the left atrium. Based on this model, the configuration of the catheter was evaluated such that the catheter makes contact with the desired points on the left atrium with constraints imposed on its motion by the transseptal puncture. The choice of the transseptal puncture location affects the uniformity of catheter manipulation. The optimal puncture location ensures maximum dexterity of the catheter within the left atrium and also ensures that the catheter has the capability of reaching various locations in the heart. Successful implementation of this algorithm in clinical practice will eventually lead to reducing the time necessary to complete the procedure, improving access to difficult regions in the left atrium, reducing the amount of manipulations of the catheter required to reach a point in the left atrium and minimizing the amount of fatigue for the clinicians.

\section{References}

1. Hansen Medical Inc., http://www.hansenmedical.com/

2. Pappone, C., Vicedomini, G., Manguso, F., Gugliotta, F., Mazzone, P., Gulletta, S., Sora, N., Sala, S., Marzi, A., Augello, G.: Robotic magnetic navigation for atrial fibrillation ablation. Journal of the American College of Cardiology 47, 1390-1400 (2006)

3. Ponti, R.D., et al.: Trans-septal catheterization in the electrophysiology laboratory: data from a multicenter survey spanning 12 years. J. Am. Coll. Cardiol. 47, 1037$1042(2006)$

4. Cannon, J., Stoll, J., Selha, S., Dupont, P., Howe, R., Torchiana, D.: Port placement planning in robot-assisted coronary artery bypass. IEEE Transactions on Robotics and Automation 19, 912-917 (2003)

5. Trejos, A.L., Patel, R.V., Ross, I., Kiaii, B.: Optimizing port placement for robotassisted minimally invasive cardiac surgery. Int. J. Med. Robotics Comput. Assist. Surg. 3, 355-364 (2007)

6. Adhami, L., Coste-Manière, È., Boissonnat, J.-D.: Planning and simulation of robotically assisted minimal invasive surgery. In: Delp, S.L., DiGoia, A.M., Jaramaz, B. (eds.) MICCAI 2000. LNCS, vol. 1935, pp. 624-633. Springer, Heidelberg (2000)

7. Badani, K.K., Muhletaler, F., Fumo, M., Kaul, S., Peabody, J.O., Bhandari, M., Menon, M.: Optimizing robotic renal surgery: The lateral camera port placement technique and current results. Journal of Endourology 22, 507-510 (2008)

8. Stocco, L., Salcudean, S.E., Sassani, F.: Fast constrained global minimax optimization of robot parameters. Robotica 16, 595-605 (1998)

9. Sciavicco, L., Siciliano, B.: A solution algorithm to the inverse kinematic problem for redundant manipulators. IEEE Transactions on Robotics and Automation 4, 403-410 (1988)

10. Merlet, J.P.: Jacobian, manipulability, condition number, and accuracy of parallel robots. Journal of Mechanical Design 128, 199-206 (2006) 